\title{
A new simple score of chronic cough: cough evaluation test
}

\author{
Wenzhi Zhan ${ }^{1 \dagger}$, Liting Zhang ${ }^{1 \dagger}$, Mei Jiang ${ }^{1 \dagger}$, Meihua Chen ${ }^{2}$, Xiaoling Yuan ${ }^{3}$, Jianxin Sun ${ }^{4}$, Pusheng Xu ${ }^{5}$, Feng Wu $\mathrm{Wu}^{6}$ \\ Chunlai Zhang ${ }^{7}$, Wei Luo ${ }^{1}$, Xiaomei Chen ${ }^{1}, \mathrm{Hu} \mathrm{Li}^{1}$ and Kefang Lai ${ }^{{ }^{*}}$
}

\begin{abstract}
Background: Chronic cough has an important impact on physical, social and psychological aspects. A simple and effective method to assess different aspects of chronic cough severity is required. We aimed to develop a simple, self-completed test, Cough Evaluation Test (CET), to evaluate cough severity and its impact on health.

Method: The items of preliminary CET were generated based on literature review and clinical practice. Items reduction was conducted by modified Delphi method. Patients with chronic cough were recruited to complete CET, Cough Visual Analog Scales (VAS), Mandarin Chinese version of the Leicester Cough Questionnaire (LCQ-MC), and Cough Symptom Score (CSS). Reassessments were performed at 1 week apart before treatment, and after more than 2 weeks treatments. Concurrent validation, internal consistency, repeatability, responsiveness and the minimal important difference (MID) were determined.

Results: CET consists of five items with a 5-point Likert scale (1-5 scaling of items, 5-25 score range). The Cronbach's alpha values for CET was 0.80. CET showed a stronger correlation with LCQ-MC $(r=-0.74)$ compared to that between LCQ-MC with VAS $(r=-0.61)$. CET also showed a stronger correlation with VAS $(r=0.70)$ compared to that between VAS with other measures. Intraclass correlation coefficients for CET was 0.84. In patients undergoing treatment, CET scores significantly changed $(p<0.0001)$. The MID of CET was 2.
\end{abstract}

Conclusion: Cough Evaluate Test is a reliable, valid and responsive tool to simply evaluate impact of cough on physical, social and psychological aspects.

Keywords: Chronic cough, Severity, Quality of life, Evaluation test, Validation

\section{Background}

Chronic cough was a common complaint of patients in respiratory clinics, which was involved in many conditions, such as cough variant asthma, eosinophilic bronchitis, atopic cough, upper airway cough syndrome and gastro-oesophageal acid reflux disease [1,2]. The global prevalence of chronic cough was up to $9.6 \%$ in general

\footnotetext{
*Correspondence: klai@163.com

Wenzhi Zhan, Liting Zhang, and Mei Jiang are Co-first authors.

${ }^{1}$ State Key Laboratory of Respiratory Disease, National Clinical Research Center for Respiratory Disease, Guangzhou Institute of Respiratory Health, the First Affiliated Hospital of Guangzhou Medical University, 151 Yanjiang Rd, Guangzhou, Guangdong 510120, P. R. China

Full list of author information is available at the end of the article
}

adult populations [3]. Chronic cough could cause significant physical, psychological and social morbidity. Besides routine clinical evaluations, reliable and valid evaluation on the impact of cough on their health status is an important step in management of the patients. An ideal measure for chronic cough should include information on cough severity, social and psychological aspects of patients and promote communication between patient and clinician. A few measurements have been used in clinical practice and clinical trials. Cough Symptom Score (CSS) was a simple two-part questionnaire relating to cough symptoms [4], which is focused on cough frequency, and overall impact on daily life. However, CSS is not easy to

(c) The Author(s). 2020 Open Access This article is licensed under a Creative Commons Attribution 4.0 International License, which permits use, sharing, adaptation, distribution and reproduction in any medium or format, as long as you give appropriate credit to the original author(s) and the source, provide a link to the Creative Commons licence, and indicate if changes were made. The images or other third party material in this article are included in the article's Creative Commons licence, unless indicated otherwise in a credit line to the material. If material is not included in the article's Creative Commons licence and your intended use is not permitted by statutory regulation or exceeds the permitted use, you will need to obtain permission directly from the copyright holder. To view a copy of this licence, visit http://creativecommons.org/licenses/by/4.0/ The Creative Commons Public Domain Dedication waiver (http://creativecommons.org/publicdomain/zero/1.0/) applies to the data made available in this article, unless otherwise stated in a credit line to the data. 
distinguish the different score level based on cough numbers, and there was little clinical experience with this tool. Cough Visual Analog Scales (VAS) is a brief subjective assessment of cough severity, but lost sight of other aspects of cough-impact on health. Available cough-specific health status measures, such as the Leicester Cough Questionnaire (LCQ) [5], and Cough-Specific Quality of-Life Questionnaire (CQLQ) [6], were reliable, valid, widely used in clinical trials. It takes more than $5 \mathrm{~min}$ to complete and calculate score of CQLQ with 28 items in six domains, and it needs scoring algorithms to calculate scores of each domain and total score of LCQ with 19 items in three domains, which makes these questionnaires are time-consuming and complex to use in clinical practice. There is a lack of a simple, effective method to assess different aspects of chronic cough in routine care [7, 8]. Therefore, we aim to develop a new simple measurement for chronic cough, Cough Evaluation Test (CET), including physical, psychological and social aspects.

\section{Method}

\section{Item generation}

Preliminary CET were first generated in reference to LCQ and clinical practice with the following criteria: 1) including three aspects of physical, psychologic, and social impact;2) integrating or subtracting similar or redundant items; 3) adding some items which are important in chronic cough management. The preliminary list of items was distributed to a Delphi expert panel. We invited 57 respiratory experts who are the fellow members of Chinese Thorax Society and specialists on field of chronic cough to evaluate the importance of items in the CET. Two Delphi rounds were conducted for comment on the list and identify further indicators. For each Delphi round, electronic surveys were used to collect the data. The Delphi group members were asked to rate the importance of each item in preliminary CET, which has 13 items on a 5-point scale $(1=$ not important, $5=$ extremely important). If the expert disagrees with the formulation of the item, explanation should be provided. For the second Delphi round, experts were asked to rate each item again, and they could change their score in view of the group's response to the previous round. Electronic reminders were sent to nonresponders 1 and 2 weeks later.

Response scale: A 5-point Likert scale was used to rate the items of CET ranging from 1 (none of the time) to 5 (all of the time.) A higher score indicated worse condition of cough. The overall score for the CET was calculated by adding the scores of each item.

\section{Validation}

\section{Subjects}

Patients with chronic cough ( $>14$ years of age) were recruited from the respiratory outpatient clinic. Chronic cough was defined as cough being sole or predominant symptom lasting more than 8 weeks, without overt identifiable abnormalities on chest X-ray. The study was approved by the Ethics Committee of the First Affiliated Hospital of Guangzhou Medical University. (Number: 201777).

\section{Reliability and item analysis}

Internal reliability was assessed by determining Cronbach's alpha coefficients which indicated the extent to which items are related. Internal reliability is generally acceptable if Cronbach's alpha coefficient is greater than 0.7. Inter-item correlations were assessed for further evidence of homogeneity and potential redundancy of items. For example, when the inter-item correlation was greater than 0.7 , it indicated that the items were similar. Items were also omitted when the item to total score correlation was less than 0.2 , indicating little contribution to the overall score. Items with ceiling or floor effect (> 50\% of the respondents chose an extreme positive or negative response category, respectively) would be removed.

\section{Concurrent validity}

Concurrent validity was assessed by correlating scores of CET with three health outcome measures completed at the same time: (1) VAS is a $100 \mathrm{~mm}$ scale on which patients indicate the severity of cough. (2) The Leicester Cough Questionnaire (LCQ) is a 19-item questionnaire that assesses cough related quality of life [5]. LCQ-MC was used and presented as its total score in this study, which had already been validated in bronchiectasis and in non-small cell lung cancer patients after surgery [9, 10]. (3) CSS is a two-part questionnaire relating to cough symptoms during the day and at night [4].

\section{Repeatability}

The test-retest procedure measured the stability of scores on CET over time in patients who had a stable condition of chronic cough. The repeatability of the CET was assessed in those patients indicating no change in cough status in 1 week apart. LCQ-MC, VAS and CSS were also completed by the subjects in the same time.

\section{Responsiveness}

Responsiveness of CET, LCQ-MC, VAS and CSS were tested before and after at least 2 weeks treatment. Individual treatment for chronic cough was based on its etiology according to the principle of cough guideline [11]. Patients were asked if their cough had improved after treatment. 
Table 1 Results for Delphi round 1 and 2

\begin{tabular}{|c|c|c|c|c|}
\hline & Round 1 & & Round 2 & \\
\hline & Mean \pm SD & Scores $\geq 4, \%$ & Mean \pm SD & Scores $\geq 4, \%$ \\
\hline 1. Cough frequency during the day & $4.35 \pm 0.55$ & $96.77 \%$ & $4.44 \pm 0.50$ & $100.00 \%$ \\
\hline 2. Cough frequency during the night & $4.58 \pm 0.56$ & $96.77 \%$ & $4.72 \pm 0.46$ & $100.00 \%$ \\
\hline 3. Cough frequency during the day and night & $4.00 \pm 1.12$ & $75.00 \%$ & $4.10 \pm 0.98$ & $82.76 \%$ \\
\hline 4. Intense cough & $4.07 \pm 0.70$ & $79.31 \%$ & $4.17 \pm 0.65$ & $86.67 \%$ \\
\hline 5. Cough beyond control & $3.79 \pm 1.15$ & $62.07 \%$ & $4.10 \pm 1.06$ & $70.00 \%$ \\
\hline 6. Cough disturbed sleep & $4.58 \pm 0.62$ & $93.55 \%$ & $4.77 \pm 0.50$ & $96.77 \%$ \\
\hline 7. Cough interfered with daily life & $4.40 \pm 0.72$ & $86.67 \%$ & $4.66 \pm 0.55$ & $96.88 \%$ \\
\hline 8. Psychological burden from cough & $3.52 \pm 0.94$ & $48.15 \%$ & $3.54 \pm 0.79$ & $53.57 \%$ \\
\hline 9. Feel anxious or depressive & $3.80 \pm 0.89$ & $63.33 \%$ & $3.87 \pm 0.72$ & $74.19 \%$ \\
\hline 10. Feel embarrassment & $3.21 \pm 0.86$ & $34.48 \%$ & $3.27 \pm 0.64$ & $36.67 \%$ \\
\hline 11. Concerned that cough was related to infectious disease or lung cancer & $2.88 \pm 1.21$ & $30.77 \%$ & $2.90 \pm 1.01$ & $27.59 \%$ \\
\hline 12. Sputum (phlegm) production & $4.58 \pm 0.72$ & $93.55 \%$ & $3.81 \pm 0.82$ & $68.75 \%$ \\
\hline 13. Difficult to expectorate & $3.84 \pm 0.82$ & $77.42 \%$ & $3.94 \pm 0.72$ & $84.38 \%$ \\
\hline
\end{tabular}

\section{Statistical analysis}

SPSS version 16 was used for data analysis. Data are presented as the mean (standard deviation) or median (range) according to its distribution. Statistical comparisons among groups were performed with one-way analysis of variance (ANOVA) for normally distributed data and Kruskal-Wallis tests for skewed data. Spearman's correlation coefficient was used to determine concurrent validity. Comparison of the magnitude of two correlations was referred to the method described previously [12]. Internal consistency and reliability were evaluated by calculating Cronbach's alpha coefficients. Repeatability testing were assessed using intraclass correlation coefficients and Bland-Altman plot. Responsiveness was analyzed by paired t-test and effect size for these measures scores that was determined by the difference in mean total the measure score before and after the treatment/SD of the measure score before the treatment. The Minimal Important Difference (MID) of CET was assessed with distribution-based method [13, 14] in which the values were calculated from the standard error of measurement (SEM). SEM $=S D \sqrt{ }(1-R)$. SD means standard deviation at baseline, and $\mathrm{R}$ means Intraclass correlation coefficients.

\section{Results}

\section{CET generation}

There were 13 items in the preliminary CET regarding physiology, social and psychological aspects. Items with physiologic aspect included cough frequency of different time (day or night), intense cough, cough beyond control, cough disturbed sleep, sputum (phlegm) production and difficulty to expectorate (Table 1). Items in regard of social aspect was presented as how cough interfered daily life, which consist of three aspects: whether cough interfered with working, daily activities, colleagues or families. Items related with psychological aspect included psychological burden, embarrassment, feeling anxious or depressive and concerning whether cough was related to infectious conditions or lung cancer. Among 57 respiratory experts who were invited to evaluate the importance of items in the CET, 33 experts responded and participated in round 1 evaluation. In the second Delphi round, experts who participated in

Table 2 Cough Evaluation Test

\begin{tabular}{|c|c|c|c|c|c|}
\hline $\begin{array}{l}\text { Please read each question carefully to assess your condition at present and answer by ' } \sqrt{ } \text { ' the } \\
\text { response that best applies to you. }\end{array}$ & $\begin{array}{l}\text { None } \\
1\end{array}$ & $\begin{array}{l}\text { Seldom } \\
2\end{array}$ & $\begin{array}{l}\text { Sometimes } \\
3\end{array}$ & $\begin{array}{l}\text { Often } \\
4\end{array}$ & $\begin{array}{l}\text { All of the } \\
\text { time } \\
5\end{array}$ \\
\hline How frequently did you cough during the day? & 1 & 2 & 3 & 4 & 5 \\
\hline Have your cough disturbed your sleep? & 1 & 2 & 3 & 4 & 5 \\
\hline Did you have intense cough? & 1 & 2 & 3 & 4 & 5 \\
\hline Have your cough interfered with your daily life? & 1 & 2 & 3 & 4 & 5 \\
\hline \multirow[t]{2}{*}{ Have your cough made you feel anxious or depressive? } & 1 & 2 & 3 & 4 & 5 \\
\hline & & & Total score: & & \\
\hline
\end{tabular}


Table 3 Baseline characteristics of the study population

\begin{tabular}{lllll}
\hline & All participants & Repeatability & Responsiveness & $p$ value \\
\hline Cases & 237 & 59 & 100 & $40.4 \pm 12.6$ \\
Age, years & $40.4 \pm 12.5$ & $37.4 \pm 11.2$ & $57 \%$ & 0.2286 \\
Gender (\% female) & $54 \%$ & $46 \%$ & $36.1 \pm 66.5$ & 0.5054 \\
Duration of cough, months & $43.0 \pm 77.3$ & $39.1 \pm 62.1$ & $13.1 \pm 3.7$ & 0.7415 \\
LCQ-MC & $12.9 \pm 3.6$ & $13.6 \pm 3.8$ & $54.4 \pm 20.8$ & 0.4777 \\
VAS, mm & $58.9 \pm 21.0$ & $52.6 \pm 20.2$ & $14.0 \pm 4.1$ & 0.2942 \\
CET & $13.9 \pm 4.2$ & $12.8 \pm 4.1$ & & 0.1534 \\
CSS & & & $2.9 \pm 0.8$ & 0.1213 \\
$\quad$ Daytime & $2.9 \pm 0.8$ & $2.7 \pm 0.8$ & $1.4 \pm 1.1$ & 0.0685 \\
Nighttime & $1.3 \pm 1.0$ & $1.0 \pm 1.0$ & $4.3 \pm 1.4$ & 0.0235 \\
Total & $4.3 \pm 1.5$ & $3.7 \pm 1.5$ & & \\
\hline
\end{tabular}

Data were presented as Mean \pm SD. LCQ-MC Mandarin Chinese version of the Leicester Cough Questionnaire, VAS Visual Analog Scales, CET Cough Evaluation Test, CSS Cough Symptom Score

previous round were asked to rate the item again. The response rate for round 2 was $93.94 \%(31 / 33)$. The mean ratings and the percentage of item-rated-score at least 4 were presented in Table 1. The top 5 items were "cough during the day", "cough during the night", "cough disturbed sleep", "cough interfered with daily life", and "intense cough". Considering the objective to evaluate of "cough disturbed sleep?" and "cough during the night" are similar, hence these two items were merged into one item. As items of CET were supposed to contain physical, psychological and social aspects of chronic cough, "feel anxious or depressive" were selected to enter the final version of CET (Table 2).

\section{Participant characteristics}

We recruited 279 chronic cough patients. Forty patients were excluded due to cough duration less than 2 months (15), poor education (7), or omission of some items of questionnaires (18). The characteristics of the remaining 237 patients were showed in Table 3. The mean age of patients was $40.4 \pm 12.5$ years, and female accounted for 128 (54\%). The scores for LCQ-MC, cough VAS, CET and CSS were also listed. After the first assessment, fiftynine patients were recruited to complete CET and other measures in 1 week apart before the treatment. And we randomly recruited 100 patients who had been assessed before and after more than 2 weeks treatment for responsiveness analysis. The causes of these 100 patients were gastro-oesophageal reflux $(n=24)$, cough variant asthma $(n=20)$, upper airway cough syndrome $(n=12)$, atopic cough $(n=8)$, chronic bronchitis $(n=7)$, bronchiectasis $(n=5)$, and other conditions $(n=24)$.

\section{Internal consistency and item analysis}

The Cronbach's alpha value of CET was 0.80 . No item had a ceiling or a floor effect. Inter-item correlations were mild to moderate (Table 4). No items were removed from this analysis as no items had consistently high or low correlations. All items had corrected itemtotal correlation coefficients greater than 0.4 and less than 0.7 (Table 5). Cronbach's alpha was high for all items, and there was no substantial increase with removal of these items, thus all items were retained.

\section{Concurrent validity}

CET showed strong correlations with VAS, LCQ-MC and CSS total scores respectively $(r=0.70, r=-0.74$, and $r=$ 0.71 , respectively). There were just mild to moderate correlation between CSS and LCQ- MC, CSS and VAS at baseline (Table 6). And the correlation between cough VAS and

Table 4 Inter-Item Correlation Matrix of the CET $(N=237)$

\begin{tabular}{|c|c|c|c|c|c|}
\hline & $\begin{array}{l}\text { Cough during } \\
\text { the day }\end{array}$ & $\begin{array}{l}\text { Cough disturbed } \\
\text { sleep }\end{array}$ & $\begin{array}{l}\text { Intense } \\
\text { cough }\end{array}$ & $\begin{array}{l}\text { Cough interfered } \\
\text { with daily life }\end{array}$ & $\begin{array}{l}\text { Feel anxious or } \\
\text { depressive }\end{array}$ \\
\hline Cough during the day & 1 & 0.27 & 0.47 & 0.43 & 0.33 \\
\hline Cough disturbed sleep & 0.27 & 1 & 0.52 & 0.46 & 0.44 \\
\hline Intense cough & 0.47 & 0.52 & 1 & 0.56 & 0.47 \\
\hline $\begin{array}{l}\text { Cough interfered with } \\
\text { daily life }\end{array}$ & 0.43 & 0.46 & 0.56 & 1 & 0.62 \\
\hline Feel anxious or depressive & 0.33 & 0.44 & 0.47 & 0.62 & 1 \\
\hline
\end{tabular}


Table 5 Item reliability for the 5 items of CET $(N=237)$

\begin{tabular}{|c|c|c|c|c|c|}
\hline & $\begin{array}{l}\text { Scale Mean if } \\
\text { Item Deleted }\end{array}$ & $\begin{array}{l}\text { Scale Variance } \\
\text { if Item Deleted }\end{array}$ & $\begin{array}{l}\text { Corrected Item-Total } \\
\text { Correlation }\end{array}$ & $\begin{array}{l}\text { Squared Multiple } \\
\text { Correlation }\end{array}$ & $\begin{array}{l}\text { Cronbach's Alpha } \\
\text { if Item Deleted }\end{array}$ \\
\hline Cough during the day & 10.35 & 13.98 & 0.47 & 0.26 & 0.80 \\
\hline Cough disturbed sleep & 11.73 & 11.64 & 0.55 & 0.33 & 0.79 \\
\hline Intense cough & 11.22 & 11.56 & 0.66 & 0.45 & 0.75 \\
\hline $\begin{array}{l}\text { Cough interfered with } \\
\text { daily life }\end{array}$ & 11.32 & 10.56 & 0.69 & 0.50 & 0.74 \\
\hline $\begin{array}{l}\text { Feel anxious or } \\
\text { depressive }\end{array}$ & 11.13 & 11.28 & 0.62 & 0.42 & 0.76 \\
\hline
\end{tabular}

LCQ-MC at baseline was $-0.61(P<0.0001)$. The strength of correlation between CET and VAS was significantly stronger than that between VAS and LCQ-MC /CSS daytime /CSS nighttime at baseline. And the strength of correlation between CET and LCQ-MS was significantly stronger than that between LCQ-MS and other measures at baseline.

There were much better correlations between CET and other outcome measures after 2 weeks treatment, compared to that at baseline (Table 7). And the correlation between VAS and LCQ-MC was - 0.76 after 2 weeks treatment. Moreover, after 2 weeks treatment, the strength of correlation between CET and LCQ-MC was significantly higher than that between LCQ-MS and other measures as well. In addition, the correlation between CET and VAS was significantly stronger than correlations between VAS and LCQ-MC /CSS total /CSS nighttime.

\section{Repeatability testing and the MID}

Intraclass correlation coefficients for CET, LCQ-MC, and VAS were high $(0.84,0.85,0.85$ respectively), while for the CSS were modest (daytime 0.64, nighttime 0.65, and total score 0.72). A Bland-Altman plot of CET score was shown in Fig. 1. The MID of CET was 2.

\section{Responsiveness testing}

Following treatment based on cough treatment guideline [11], CET, VAS, LCQ-MC and CSS scores changed $(p<$ 0.0001 , Table 8 ). The effect sizes for change of these measures were presented in Table 8. There were strong relationship between change in CET score and that in VAS score $(r=0.72, p<0.0001)$, LCQ scores $(r=-0.70$, $P<0.0001)$, and CSS total scores $(r=0.66, P<0.0001)$.

\section{Discussion}

This study has created a short, simple patient-completed test for chronic cough patients with good measurement properties. Almost all patients could complete the CET within 1 minute in this study. The 5 items selected for CET parallel the dimensions of cough managementcough severity, social impact and psychological effect. In this study, scores computed from CET were shown to be highly repeatable and responsive, suggesting CET may be a useful outcome measure in assessing the response to intervention in clinical practices and trials.

Cronbach's alpha coefficient of CET was 0.80 , which was sufficient in internal consistency. No matter at baseline or after treatment, CET has shown a strong correlation with cough VAS and LCQ-MC, and its correlation intensity were significantly higher than that between cough VAS and LCQ-MC, and were also higher than those between cough VAS or LCQ-MC and other measures, indicating CET was useful to assess cough severity and cough-related quality of life. Although intraclass correlation coefficient of LCQ-CM was lower than that in the previous researches $(0.89-0.96)[5,9,10]$, but it still has excellent level of repeatability. The intraclass

Table 6 Spearman rank correlations between different health outcome measures at baseline $(N=237)$

\begin{tabular}{|c|c|c|c|c|c|c|}
\hline & CET & VAS & LCQ-MC & CSS Total & CSS daytime & CSS nighttime \\
\hline$\overline{\text { CET }}$ & & $0.70^{* \#}$ & $-0.74^{* \&}$ & $0.71^{*}$ & $0.60^{*}$ & $0.53^{*}$ \\
\hline VAS & $0.70^{* \#}$ & & $-0.61^{*}$ & $0.66^{*}$ & $0.60^{*}$ & $0.43^{*}$ \\
\hline LCQ-MC & $-0.74^{* \&}$ & $-0.61^{*}$ & & $-0.54^{*}$ & $-0.50^{*}$ & $-0.38^{*}$ \\
\hline CSS Total & $0.71^{*}$ & $0.66^{*}$ & $-0.54^{*}$ & & $0.76^{*}$ & $0.79 *$ \\
\hline CSS daytime & $0.60^{*}$ & $0.60^{*}$ & $-0.50^{*}$ & $0.76^{*}$ & & $0.23^{*}$ \\
\hline CSS nighttime & $0.53^{*}$ & $0.43^{*}$ & $-0.38^{*}$ & $0.79^{*}$ & $0.23^{*}$ & \\
\hline
\end{tabular}

\section{*P<0.0001}

\# correlation between CET and cough VAS versus correlation between VAS and LCQ-MS or correlation between VAS and CSS daytime/nighttime $(P<0.0001, P=0.0143$, $P<0.0001$, respectively)

\& correlation between CET and LCQ-MC versus correlation between VAS and LCQ-MS or correlation between LCQ-MS and CSS total/daytime/nighttime $(P=0.0002$, $P<0.0001, P<0.0001, P<0.0001$, respectively) 
Table 7 Spearman rank correlations between different health outcome measures after treatment $(N=100)$

\begin{tabular}{|c|c|c|c|c|c|c|}
\hline & CET & VAS & LCQ-MC & CSS Total & CSS daytime & CSS nighttime \\
\hline$\overline{\mathrm{CET}}$ & & $0.87^{* \#}$ & $-0.85^{* \&}$ & $0.75^{*}$ & $0.78^{*}$ & $0.49^{*}$ \\
\hline VAS & $0.87^{* \#}$ & & -0.76 & $0.78^{*}$ & $0.82^{*}$ & $0.45^{*}$ \\
\hline LCQ-MC & $-0.85^{* \&}$ & $-0.76^{*}$ & & $-0.76^{*}$ & $-0.72^{*}$ & $-0.56^{*}$ \\
\hline CSS Total & $0.75^{*}$ & $0.78^{*}$ & $-0.76^{*}$ & & $0.84^{*}$ & $0.78^{*}$ \\
\hline CSS daytime & $0.78^{*}$ & $0.82^{*}$ & $-0.72^{*}$ & $0.84^{*}$ & & $0.37^{*}$ \\
\hline CSS nighttime & $0.49^{*}$ & $0.45^{*}$ & $-0.56^{*}$ & $0.78^{*}$ & $0.37^{*}$ & \\
\hline
\end{tabular}

* $P<0.0001$

\# correlation between CET and VAS versus correlation between VAS and LCQ-MS or correlation between VAS and CSS total/nighttime $(P<0.0001, P=0.0082$, $P<0.0001$, respectively)

\& correlation between CET and LCQ-MC versus correlation between VAS and LCQ-MS or correlation between LCQ-MS and CSS total/daytime/nighttime $(P=0.0015$, $P=0.0145, P=0.0004, P<0.0001$, respectively)

correlation coefficient of cough VAS $(0.85)$ was almost the same as Birring's data (0.84) [5], indicating VAS also has a stable and excellent test-retest reliability. In addition, CET has the same excellent degree of testretest reliability as LCQ-MC and cough VSA did, and Bland-Altman plot of CET also showed its excellent repeatability.

Our data also showed that the CET was responsive to change after treatment and the effect size was less than that seen with the cough VAS, but was more than that seen with LCQ-MC. Cohen proposed benchmarks that serve to guide the interpretation of effect sizes: 0.2 for 'small' effects, 0.5 for 'medium' effects, and 0.8 for 'large' effects [15]. In our study, CET and cough VAS can detect "large" effects, while LCQ-MC can just reflect the "medium" effect after the treatment, suggesting that CET and cough VAS might be better outcome measures of choice in clinical trials. The effect size represents individual change in terms of the number of pre-test SD, which mean that characteristics of the distribution, particularly at baseline, may strongly influence the effect

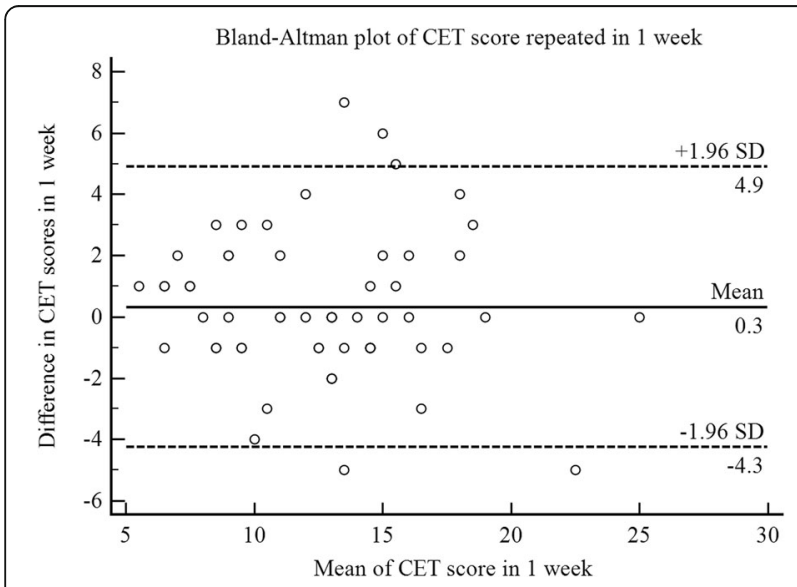

Fig. 1 Bland-Altman plot of CET score repeated in 1week in 59 patients with chronic cough. $---=$ mean difference between the two scores; - - - = 95\% limits of agreement size [13]. And it could explain why effect sizes in our data were less than that in Birring' result [5].

More than $80 \%$ of cough occurs during awake time [16], while some patients like cough variant asthma may also suffer from nocturnal cough. One direct effect of nocturnal cough was sleep disturbance. Moreover, cough intensity was also an important determinant of cough severity in some patients $[16,17]$. Three items of CET including "How frequently did you cough during the day?", "Have your cough disturbed your sleep?", "Did you have intense cough?" correspond to cough severity that was a single concept with three inter-related components: frequency, intensity, and disruption [18]. Cough is protective reflex. Some smokers may cough a lot, but that may not be a big deal for them, while some patients who are social or have to give a speech frequently, coughing may be a huge issue. In addition to physical discomfort, a protracted cough could also cause anxiety, social and personal embarrassment, and deterioration in quality of life especially in psychosocial condition [19, 20], which was also reflected in the last two items of CET with "Have your cough interfered with your daily life?", and "Have your cough made you feel anxious or depressive?" . The CET correlated well with cough VAS and LCQ-MC, suggesting its great capability of assessing cough severity and cough-specific quality of-life. However, for chronic cough patients, especially for those who were diagnosed as unexplained or refractory chronic cough whose pathogenesis is inadequately understood and effective treatment is still lacking, whether CET is appropriated tool for long-term management and which cutoff score of CET is appropriate to identify the need of drug therapy still require further research.

Koo, Hyeon-Kyoung et al. have described the validation of another cough assessment test (COAT) [21]. Unlike the full evaluation of CET on cough severity, social impact and psychological effect, the COAT focuses on cough frequency, limitation on daily activities, sleep disturbance, fatigue and hypersensitivity to irritants, no sight of cough intensity and psychological impact. 
Table 8 Comparison of pre-post treatment CET, LCQ-MC, VAS and CSS scores in the responsiveness component of the study $(n=$ 100)

\begin{tabular}{lllllll}
\hline & CET & LCQ-MC & VAS & CSS total & CSS daytime & CSS nighttime \\
\hline Pre-treatment & $14.0(4.1)$ & $13.1(3.7)$ & $54.4(20.8)$ & $4.3(1.4)$ & $2.9(0.8)$ & $1.4(1.1)$ \\
Post-treatment & $10.2(3.9)$ & $15.6(3.7)$ & $32.2(22.0)$ & $2.8(1.6)$ & $1.9(1.2)$ & $0.9(0.9)$ \\
$P$ value for paired t-test & $P<0.0001$ & $P<0.0001$ & $P<0.0001$ & $P<0.0001$ & $P<0.0001$ & $P<0.0001$ \\
Effect sizes & 0.9 & 0.7 & 1.1 & 1.1 & 1.2 & 0.5 \\
\hline
\end{tabular}

Data are presented as mean (SD)

Further work is necessary to compare the CET and COAT in the evaluation of chronic cough.

There are limitations to our study. The MID of CET was just based on distribution-based method. Several studies have showed that a MID based on anchor-based approach was close to the value of one SEM [22, 23], while a few discrepancies were showed in other researches $[24,25]$. It seems that one SEM equals the MID is not a universal truth [26]. In addition, the subjects selected for the test validation were older than 14 years without overt identifiable abnormalities on chest X-ray, which may restrict its application in children and those with abnormal chest radiograph.

\section{Conclusion}

We develop a new cough evaluation test consisting 5 items. Through assessment with concurrent validation, internal consistency, repeatability, responsiveness and the minimal important difference, CET is a reliable, valid and responsive tool to simply evaluate full impact of chronic cough in regard of physical, social and psychological aspects. CET would facilitate an easy and efficient way to assess chronic cough in routine care and clinical trials.

\section{Abbreviations}

CET: Cough Evaluation Test; COAT: Cough assessment test; CSS: Cough Symptom Score; LCQ: Leicester Cough Questionnaire; LCQ-MC: Mandarin Chinese version of the Leicester Cough Questionnaire; MID: The minimal important difference; VAS: Visual analog scales

\section{Acknowledgements}

The authors thank all the patients who participated in this study and Professor Thys van der Molen for professional writing suggestion. The authors also thank all the experts of Delphi expert panel.

\section{Authors' contributions}

All authors participated in manuscript writing and editing. Conception and design: KL. Recruiting subjects and acquisition of data: $W Z, L Z, M J, M C, X Y$, JS, PX, FW CZ, WL, XC, HL. Analysis and interpretation: WZ, LZ, MJ. Critical review and editing: $K L, W Z, L Z, M J$. All authors read and approved the final manuscript.

\section{Funding}

This study was supported by National Natural Science Foundation of China (81570092); Guangzhou Municipal Science and Technology Key Project(2002Z2E0091); Incubative Project for Innovation Team of GMU(2017-159); and Clinical Research Foundation of GMU(2017-160). The funders had no role in study design, data collection and analysis, decision to publish, or preparation of the manuscript.

\section{Availability of data and materials}

The datasets used and/or analyzed during the current study are available from the corresponding author on reasonable request.

\section{Ethics approval and consent to participate}

The study was approved by the research ethics committee of the First Affiliated Hospital of Guangzhou Medical University. (No. 201777) All subjects gave written informed consents prior to participation.

Consent for publication

Not applicable.

\section{Competing interests}

The authors declare that they have no competing interests.

\section{Author details}

${ }^{1}$ State Key Laboratory of Respiratory Disease, National Clinical Research Center for Respiratory Disease, Guangzhou Institute of Respiratory Health, the First Affiliated Hospital of Guangzhou Medical University, 151 Yanjiang Rd, Guangzhou, Guangdong 510120, P. R. China. ${ }^{2}$ Respiratory Department of the Third People's Hospital of Dongguan city, Dongguan, Guangdong, P. R. China. 'hongshan Hospital of Sun Yat-sen University, Zhongshan People's Hospital, Zhongshan, Guangdong, P. R. China. ${ }^{4}$ The Second People's Hospital of Zhaoqing, Zhaoqing, Guangdong, P. R. China. ${ }^{5}$ Department of Respiratory, The Second Affiliated Hospital of Guangzhou Medical University, Guangzhou, Guangdong, P. R. China. ${ }^{6}$ Huizhou Third People's Hospital, Guangzhou Medical University, Huizhou, Guangdong, P. R. China. ${ }^{7}$ Jiangmen Central Hospital, Jiangmen, Guangdong, P. R. China.

Received: 3 December 2019 Accepted: 2 March 2020

Published online: 20 March 2020

\section{References}

1. Lai K, Chen R, Lin J, Huang K, Shen H, Kong L, Zhou X, Luo Z, Yang L, Wen $F$, et al. A prospective, multicenter survey on causes of chronic cough in China. Chest. 2013;143(3):613-20.

2. Chung KF, Pavord ID. Prevalence, pathogenesis, and causes of chronic cough. Lancet. 2008;371(9621):1364-74.

3. Song W, Chang Y, Faruqi S, Kim J, Kang M, Kim S, Jo E, Kim M, Plevkova J, Park $\mathrm{H}$, et al. The global epidemiology of chronic cough in adults: a systematic review and meta-analysis. Eur Respir J. 2015;45(5):1479-81.

4. Hsu JY, Stone RA, Logan-Sinclair RB, Worsdell M, Busst CM, Chung KF. Coughing frequency in patients with persistent cough: assessment using a 24 hour ambulatory recorder. Eur Respir J. 1994;7(7):1246-53.

5. Birring SS, Prudon B, Carr AJ, Singh SJ, Morgan MD, Pavord ID. Development of a symptom specific health status measure for patients with chronic cough: Leicester cough questionnaire (LCQ). Thorax. 2003;58(4):339-43.

6. French $C T$, Irwin RS, Fletcher KE, Adams TM. Evaluation of a cough-specific quality-of-life questionnaire. CHEST. 2002;121(4):1123-31.

7. Brignall $K$, Jayaraman B, Birring SS. Quality of life and psychosocial aspects of cough. Lung. 2008;186(S1):55-8.

8. Birring SS, Spinou A. How best to measure cough clinically. Curr Opin Pharmacol. 2015;22:37-40

9. Gao Y, Guan W, Xu G, Gao Y, Lin Z, Tang Y, Lin Z, Li H, Luo Q, Zhong N, et al. Validation of the mandarin Chinese version of the Leicester cough questionnaire in bronchiectasis. Int J Tuberc Lung Dis. 2014;18(12):1431-7. 
10. Lin $\mathrm{R}$, Che $\mathrm{G}$. Validation of the mandarin Chinese version of the Leicester cough questionnaire in non-small cell lung cancer patients after surgery. Thorac Cancer. 2018;9(4):486-90.

11. Asthma Workgroup of Chinese Society of Respiratory Diseases (CSRD), Chinese Medical, Association. The Chinese national guidelines on diagnosis and management of cough (2015). Chin J Tuberc Respir Dis. 2016;39(5):32354.

12. Diedenhofen B, Musch J. cocor: a comprehensive solution for the statistical comparison of correlations. PLoS One. 2015;10(4):e121945.

13. Wyrwich KW, Tierney WM, Wolinsky FD. Further evidence supporting an SEM-based criterion for identifying meaningful intra-individual changes in health-related quality of life. J Clin Epidemiol. 1999;52(9):861-73.

14. Crosby RD, Kolotkin RL, Williams GR. Defining clinically meaningful change in health-related quality of life. J Clin Epidemiol. 2003;56(5):395-407.

15. Cohen J. A power primer. Psychol Bull. 1992;112(1):155-9.

16. Birring SS. Controversies in the evaluation and management of chronic cough. Am J Resp Crit Care. 2011;183(6):708-15.

17. Lee KK, Ward K, Rafferty GF, Moxham J, Birring SS. The intensity of voluntary, induced, and spontaneous cough. Chest. 2015;148(5):1259-67.

18. Vernon M, Leidy NK, Nacson A, Nelsen L. Measuring cough severity: perspectives from the literature and from patients with chronic cough. Cough. 2009;5:5.

19. Iyer VN, Lim KG. Chronic cough: an update. Mayo Clin Proc. 2013;88(10): $1115-26$.

20. French CL, Irwin RS, Curley FJ, Krikorian CJ. Impact of chronic cough on quality of life. Arch Intern Med. 1998;158(15):1657-61.

21. Koo H, Jeong I, Kim J, Kim S, Shin J, Park SY, Rhee CK, Choi EY, Moon J, Kim $\mathrm{YH}$, et al. Development and validation of the COugh assessment test (COAT). Respirology. 2019;24(6):551-7.

22. Wyrwich KW, Nienaber NA, Tierney WM, Wolinsky FD. Linking clinical relevance and statistical significance in evaluating intra-individual changes in health-related quality of life. Med Care. 1999;37(5):469-78.

23. Cella D, Eton DT, Fairclough DL, Bonomi P, Heyes AE, Silberman C, Wolf MK, Johnson $\mathrm{DH}$. What is a clinically meaningful change on the functional assessment of Cancer therapy-lung (FACT-L) questionnaire?: results from eastern cooperative oncology group (ECOG) study 5592. J Clin Epidemiol. 2002:55(3):285-95

24. Yost KI, Cella D, Chawla A, Holmgren E, Eton DT, Ayanian JZ, West DW. Minimally important differences were estimated for the functional assessment of Cancer therapy-colorectal (FACT-C) instrument using a combination of distribution- and anchor-based approaches. J Clin Epidemiol. 2005;58(12):1241-51.

25. Wyrwich KW. Minimal important difference thresholds and the standard error of measurement: is there a connection? J Biopharm Stat. 2004;14(1): 97-110.

26. de Vet HC, Terwee CB, Ostelo RW, Beckerman H, Knol DL, Bouter LM. Minimal changes in health status questionnaires: distinction between minimally detectable change and minimally important change. Health Qual Life Outcomes. 2006;:54

\section{Publisher's Note}

Springer Nature remains neutral with regard to jurisdictional claims in published maps and institutional affiliations.

Ready to submit your research? Choose BMC and benefit from:

- fast, convenient online submission

- thorough peer review by experienced researchers in your field

- rapid publication on acceptance

- support for research data, including large and complex data types

- gold Open Access which fosters wider collaboration and increased citations

- maximum visibility for your research: over $100 \mathrm{M}$ website views per year

At $\mathrm{BMC}$, research is always in progress.

Learn more biomedcentral.com/submissions 